\title{
A REPAIR REPLACEMENT MODEL
}

\author{
LAM YEH, ${ }^{*}$ The Chinese University of Hong Kong
}

\begin{abstract}
In this paper, we study a similar replacement model in which the successive survival times of the system form a process with non-increasing means, whereas the consecutive repair times after failure constitute a process with non-decreasing means. The system is replaced at the time of the $N$ th failure since the installation or last replacement. Based on the long-run average cost per unit time, we determine the optimal replacement policy $N^{*}$ and the maximum of the long-run average reward explicitly. Under additional conditions, the policy $N^{*}$ is even optimal among all replacement policies.
\end{abstract}

IFR; NBUE; RENEWAL THEORY

\section{Introduction}

At an early stage, the development of the replacement model is based on the assumption that a failure system after repair will yield a functioning system which is 'as good as new'. However, this model is no longer applicable to a repairable deteriorating system. For instance, in machine maintenance problems, in view of ageing and cumulative wear, on the one hand, the operating times of a machine after repair will usually become shorter and shorter and may tend to zero; on the other hand, the repair times will become longer and longer, sometimes even tending to infinity, i.e., finally the machine will be non-repairable. Thus, an appropriate model for these problems might be as follows: the successive survival times are stochastically decreasing and the consecutive repair times are stochastically increasing.

Downton (1971) and Thompson (1981) have used the non-homogeneous Poisson process for modelling these deteriorating systems. This is in fact the minimal repair model (see Barlow and Proschan (1965) or Ascher and Feingold (1984)). Another possible approach is to consider some kind of monotone process. Lam (1988a, b) studied a replacement model in which the successive survival times of the system form a non-increasing geometric process and the consecutive repair times constitute a non-decreasing geometric process.

Stadje and Zuckerman (1990) consider a more general repair replacement model by making the following assumptions: the successive survival times $\left\{X_{n}, n=1,2, \ldots\right\}$ of the system form a stochastically decreasing process and each survival time has a non-decreasing failure rate (IFR), also the consecutive repair times $\left\{Y_{n}, n=1,2, \ldots\right\}$ constitute a stochastically increasing process and each repair time has the property that new is better than used in expectation (NBUE). A replacement policy $T$ is used, by which we replace the system at time $T$ where $T$ is the time elapsed since the installation or the last replacement.

Assume that the repair cost rate is $C$. The replacement cost during an operating interval is $C_{0}$. Also, if the system is replaced upon failure or during repair, the replacement cost is assumed to be $C_{f}$ and $C_{f} \geqq C_{0}$. Whenever the system is operating, the reward rate is $R>0$. Then, we have the following result.

Received 12 September 1989; revision received 30 January 1990.

* Postal address: Department of Statistics, The Chinese University of Hong Kong, Shatin, N.T., Hong Kong. 
Theorem 1 (Stadje and Zuckerman (1990)). Suppose that:

(i) For each $n \geqq 1, X_{n}$ and $Y_{n}$ are both non-negative random variables, also $\lambda_{n}=E\left(X_{n}\right)$ is non-increasing and $\mu_{n}=E\left(Y_{n}\right)$ is non-decreasing;

(ii) $\lim _{n \rightarrow \infty} \lambda_{n}=0$ or $\lim _{n \rightarrow \infty} \mu_{n}=\infty$;

(iii) $\left\{X_{n}, n=1,2, \ldots\right\}$ and $\left\{Y_{n}, n=1,2, \ldots\right\}$ are two independent sequences of independent random variables, also for each $n \geqq 1, X_{n}$ has IFR and $Y_{n}$ is NBUE;

(iv) $C_{0}=C_{f}$.

Then the replacement policy

is optimal, where

$$
T^{*}=\sum_{i=1}^{n_{0}} X_{i}+\sum_{i=1}^{n_{0}-1} \dot{Y}_{i}
$$

$$
n_{0}=\min \left\{n \geqq 1 \mid\left(C+\varphi^{*}\right) \mu_{n} \geqq\left(R-\varphi^{*}\right) \lambda_{n+1}\right\}
$$

and $\varphi^{*}$ is the optimal value of the long-run average reward.

Thus, under some mild conditions, for a repairable deteriorating system, the optimal replacement policy is of the form 'replace after the $N$ th failure', which we call policy $N$. To determine the optimal policy $T^{*}$, by Theorem 1 we have to specify $n_{0}$ which depends on $\psi^{*}$. However, $\psi^{*}$ is usually unknown before the optimal policy $T^{*}$ has been determined. To overcome this difficulty and solve the problem completely, a direct way is to find the optimal replacement policy $N^{*}$, then $T^{*}$ and $\psi^{*}$ can be evaluated immediately.

In this paper, we generalize Lam's earlier work $(1988 \mathrm{a}, \mathrm{b})$ by introducing a model for a repairable deteriorating system which is more general than the model suggested by Stadje and Zuckerman (1990). In our model we study only the replacement policy $N$. Based on an explicit expression of the long-run average reward per unit time, we determine the optimal replacement policy $N^{*}$ and the corresponding optimal value of the long-run average reward $\psi^{*}$ explicitly.

\section{Model}

We consider the following repair replacement model.

Assumption 1. At the beginning, a new system is installed. Whenever the system fails, it is either repaired or replaced by a new and identical one.

Assumption 2. Let $X_{k}$ be the survival time after the $(k-1)$ th repair, then $\left\{X_{k}\right.$, $k=1,2, \ldots\}$ forms a sequence of non-negative random variables with non-increasing means $E\left(X_{k}\right)=\lambda_{k}$.

Assumption 3. Let $Y_{k}$ be the repair time after the $k$ th failure, then $\left\{Y_{k}, k=1,2, \ldots\right\}$ constitutes a sequence of non-negative random variables with non-decreasing means $E\left(Y_{k}\right)=$ $\mu_{k}$.

Assumption 4. The system will be replaced by a replacement policy $N$.

Assumption 5. The repair cost rate is $C$, the replacement cost under policy $N$ is $C_{f}$ and the reward rate whenever the system is operating is $R$. By classical arguments from renewal theory, the long-run average reward per unit time is given by

$$
\begin{aligned}
R_{F}(N) & =\frac{R \sum_{k=1}^{N} \lambda_{k}-C \sum_{k=1}^{N-1} \mu_{k}-C_{f}}{\sum_{k=1}^{N} \lambda_{k}+\sum_{k=1}^{N-1} \mu_{k}} \\
& =R-C_{F}(N)
\end{aligned}
$$


where

$$
C_{F}(N)=\frac{(C+R) \sum_{k=1}^{N-1} \mu_{k}+C_{f}}{\sum_{k=1}^{N} \lambda_{k}+\sum_{k=1}^{N-1} \mu_{k}}, \quad N=1,2, \ldots
$$

Then, the optimal replacement policy $N^{*}$ can be determined by maximizing $R_{F}(N)$ or minimizing $C_{F}(N)$. To do this, without loss of generality assume that $\left\{\lambda_{k}, k=1,2, \ldots\right\}$ and $\left\{\mu_{k}, k=1,2, \ldots\right\}$ are all positive.

Now, starting from (3), straightforward calculation yields

$$
C_{F}^{*}(N+1)-C_{F}^{*}(N)=\left\{(C+R) f_{N}-C_{f}\left(\lambda_{N+1}+\mu_{N}\right)\right\} / \Delta_{N}
$$

where

and

$$
\Delta_{n}=\left(\sum_{k=1}^{N+1} \lambda_{k}+\sum_{k=1}^{N} \mu_{k}\right)\left(\sum_{k=1}^{N} \lambda_{k}+\sum_{k=1}^{N-1} \mu_{k}\right)
$$

$$
f_{N}=\mu_{N} \sum_{k=1}^{N} \lambda_{k}-\lambda_{N+1} \sum_{k=1}^{N-1} \mu_{k} \text {. }
$$

Note that $\Delta_{n}>0$ and $f_{N} \geqq \dot{\lambda}_{1} \mu_{N}>0$ for all $N \geqq 1$.

Then, define $g_{N}=\left(\lambda_{N+1}+\mu_{N}\right) / f_{N}$, hence

$$
g_{N+1}-g_{N}=\left(\lambda_{N+2} \mu_{N}-\lambda_{N+1} \mu_{N+1}\right)\left(\sum_{k=1}^{N+1} \lambda_{k}+\sum_{k=1}^{N} \mu_{k}\right) /\left(f_{N} f_{N+1}\right) \leqq 0,
$$

and we have the following result.

Lemma 1. $\left\{g_{N}\right\}$ is a non-increasing sequence from $g_{1}=\left(\lambda_{2}+\mu_{1}\right) /\left(\lambda_{1} \mu_{1}\right)$ to $g_{\infty}=\lim _{N \rightarrow \infty} g_{N}$.

The next lemma follows from (3).

Lemma 2.

$$
C_{F}(N+1) \gtreqless C_{F}(N) \text { iff } g_{N} \gtreqless(C+R) / C_{f} .
$$

The combination of Lemma 1 and 2 yields the following result.

Theorem 2. The optimal policy $N^{*}$ is determined by

$$
N^{*}=\min \left\{N \geqq 1 \mid g_{N} \leqq(C+R) / C_{f}\right\} .
$$

If besides Assumptions 1-5, the conditions (ii)-(iv) of Theorem 1 also hold, then the optimal replacement policy $N^{*}$ given by (6) is exactly the same as $T^{*}$ given by (1), hence $N^{*}$ is also optimal among all policies $T$. By (6), we can find $N^{*}$; then from (2),

$$
\psi^{*}=R_{F}\left(N^{*}\right)=R-C_{F}\left(N^{*}\right) \text {. }
$$

Note that, by (6), $N^{*}$ is non-increasing in $C$ and non-decreasing in $C_{f}$.

\section{Comments}

This model assumes neither the independence of successive survival times or that of consecutive repair times, nor independence between them. Consequently, it is a realistic and practical model, because in applications, we do not need to consider and test for independence.

Essentially, the model requires only monotonicity in the means $\lambda_{n}$ and $\mu_{n}$. Moreover, (2) and (3) show that $R_{F}(N)$ and $C_{F}(N)$ depend on the processes $\left\{X_{n}, n=1,2, \ldots\right\}$ and $\left\{Y_{n}\right.$, $n=1,2, \ldots\}$ only through parameters $\lambda_{n}$ and $\mu_{n}$ and are independent of their true distributions. Of course, this distribution-free property has more extensive applications in practice. Thus, the model is a very general one.

The replacement policy $N$ has been considered by Morimura (1970) and Nakagawa (1984). 
It is an effective policy because the total operating time of a system is not recorded. For practical purposes, the implementation of the optimal policy and hence the management of the model are quite simple.

Under some mild additional conditions (Conditions (ii)-(iv) in Theorem 1), the optimal policy $N^{*}$ is also optimal among all policies $T$.

\section{Acknowledgement}

The author is indebted to the referee for his valuable suggestions which considerably improved the presentation.

\section{References}

Ascher, H. AND Feingold, H. (1984) Repairable Systems Reliability. Marcel Dekker, New York. BARLow, R. E. AND Proschan, F. (1965) Mathematical Theory of Reliability. Wiley, New York.

Downton, F. (1971) Stochastic models for successive failures. Proc. 38th Session Internat. Statist. Inst. 44 (1), 667-694.

Lam Yeh (1988a) A note on the optimal replacement problem. Adv. Appl. Prob. 20, 479-482.

LaM YeH (1988b) Geometric process and replacement problem. Acta Math. Appl. Sinica 4, 366-377.

MORIMURA, H. (1970) On some preventive maintenance policies for IFR. J. Operat. Res. Soc. Japan 12, 94-124.

NaKagawa, T. (1984) Discrete Replacement Models. Stochastic Models in Reliability. Lecture Notes in Economics and Mathematical Systems 235, Springer-Verlag, Berlin.

STADJE, W. AND ZuCKeRMAN, D. (1990) Optimal strategies for some repair replacement models. Adv. Appl. Prob. 22 (3).

THOMPSON, W. A. (1981) On the foundations of reliability. Technometrics 23, 1-13. 